\title{
Statistical gamma-ray decay studies at iThemba LABS
}

\author{
M. Wiedeking, ${ }^{1, a}$, L.A. Bernstein ${ }^{2,3}$, D.L. Bleuel ${ }^{4}$, C.P. Brits ${ }^{1,5}$, K. Sowazi ${ }^{1,6}$, A. Görgen ${ }^{7}$ B.L. Goldblum ${ }^{3}$, \\ M. Guttormsen ${ }^{7}$, B.V. Kheswa ${ }^{7}$, A.C. Larsen ${ }^{7}$, S.N.T. Majola ${ }^{1,5}$, K.L. Malatji ${ }^{1,6}$, D. Negi ${ }^{1,8}$, T. Nogwanya ${ }^{1,6}$, S. Siem $^{7}$, \\ and B.R. Zikhali ${ }^{1,9}$ \\ 1 iThemba LABS, PO Box 722, Somerset West 7129, South Africa \\ 2 Lawrence Berkeley National Laboratory, Berkeley, California 94720, USA \\ ${ }^{3}$ University of California, Berkeley, California 94720-1730, USA \\ ${ }^{4}$ Lawrence Livermore National Laboratory, Livermore, California 94550-9234, USA \\ 5 Stellenbosch University, Matieland 7602, South Africa \\ ${ }^{6}$ University of the Western Cape, Bellville 7535, South Africa \\ 7 University of Oslo, 0316 Oslo, Norway \\ ${ }^{8}$ UM-DAE Centre for Excellence in Basic Sciences, Mumbai 400098, India \\ 9 University of Zululand, KwaDlangezwa 3886, South Africa
}

\begin{abstract}
A program to study the $\gamma$-ray decay from the region of high-level density has been established at iThemba LABS, where a high-resolution gamma-ray detector array is used in conjunction with silicon particle-telescopes. Results from two recent projects are presented: 1) The ${ }^{74} \mathrm{Ge}\left(\alpha, \alpha^{\prime} \gamma\right)$ reaction was used to investigate the Pygmy Dipole Resonance. The results were compared to $\left(\gamma, \gamma^{\prime}\right)$ data and indicate that the dipole states split into mixed isospin and relatively pure isovector excitations. 2) Data from the ${ }^{95} \mathrm{Mo}(\mathrm{d}, \mathrm{p})$ reaction were used to develop a novel method for the determination of spins for low-lying discrete levels utilizing statistical $\gamma$-ray decay in the vicinity of the neutron separation energy. These results provide insight into the competition of $(\gamma, \mathrm{n})$ and $\left(\gamma, \gamma^{\prime}\right)$ reactions and highlights the need to correct for angular momentum barrier effects.
\end{abstract}

\section{Introduction}

An incredible wealth of information can be obtained from experimental investigations of the low-energy tail of the Giant Electric Dipole Resonance. The Photon Strength Function (PSF), which represents the ability of nuclear matter to absorb and emit photons, is one of the quantities that can be successfully used to extract information in the region of the quasi-continuum. However, it is becoming increasingly apparent that no single measurement can provide sufficient insight to fully understand the observed features of the PSF. Instead, most of the knowledge of the underlying nuclear structure is obtained by combining results from several experimental approaches and techniques. Over the last decade, significant experimental effort has been invested to study the $\gamma$-ray decay from the region of high-level density below the particle separation energies at many laboratories worldwide. Coordinated measurements have taken place, selecting specific nuclei and extracting the PSF through several experimental methods or beam/target combinations.

The PSF obtained from different measurements often exhibit significant discrepancies in the data. For example, the mismatch between the PSF from $\left(\gamma, \gamma^{\prime}\right)$ and chargedparticle measurements has been a point of contention for several years, e.g. Refs. [1-3]. As more results

a e-mail: wiedeking@tlabs.ac.za become available the problem is compounded due to the discrepancies not being consistently observed. For instance, analytical work at iThemba LABS to extract the PSF of ${ }^{91} \mathrm{Zr}$ using the Oslo method [4], agrees with ${ }^{90} \operatorname{Zr}\left(\gamma, \gamma^{\prime}\right)$ [5] data, within the uncertainties, as shown in Fig. 1. Recently, excellent agreement between the PSFs from charged particle and $\left(\gamma, \gamma^{\prime}\right)$ induced reactions have also been observed in ${ }^{74} \mathrm{Ge}$ [7], shown in Fig. 2. With no obvious resolution available to explain the underlying reasons for the agreement or inconsistency of data, the following questions are often raised: i) Do different experimental techniques yield the same results? ii) Do different reactions give the same PSFs? iii) Are data sets always properly normalized? iv) Are all sources of uncertainties properly treated?

In this contribution, the experimental work on ${ }^{74} \mathrm{Ge}$ and ${ }^{95} \mathrm{Mo}$ are discussed in the context of these questions and how these can only be resolved through coordinated research. ${ }^{74} \mathrm{Ge}$ and ${ }^{95} \mathrm{Mo}$ are two of the nuclei that have triggered broad collaborative efforts between several institutions, by coordinating research projects to obtain best possible knowledge of the PSFs of theses nuclei. These two cases, for which significant experimental and analytical effort has been invested at iThemba LABS, makes them excellent cases to investigate consistencies and/or differences between various experimental data. The successes and difficulties that were encountered when comparing data from different techniques are discussed. 


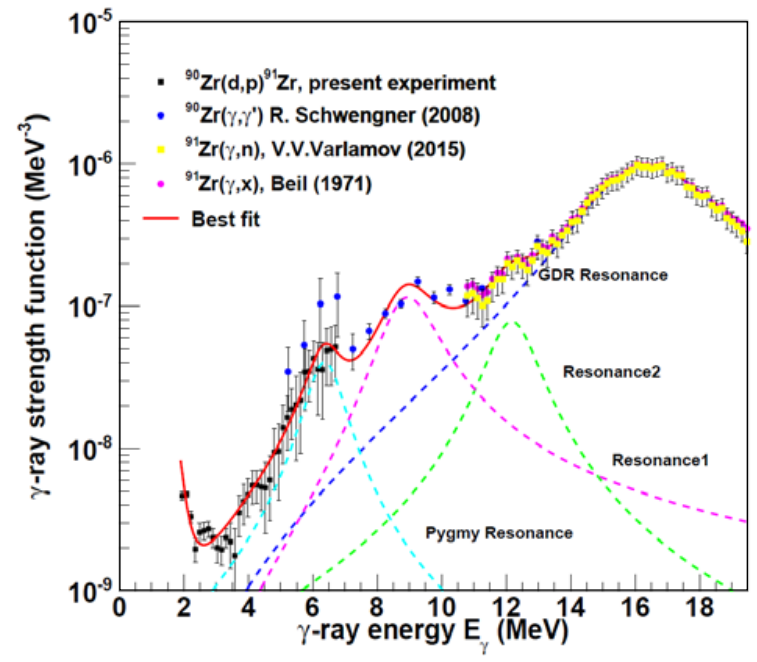

Figure 1. The photon-strength function of ${ }^{91} \mathrm{Zr}$ from data analysed at iThemba LABS using the Oslo method [4] and from ${ }^{90} \operatorname{Zr}\left(\gamma, \gamma^{\prime}\right)$ [5]. The best fit to the photon strength function is indicated by the red line and includes $(\gamma, \mathrm{x})$ data [6].

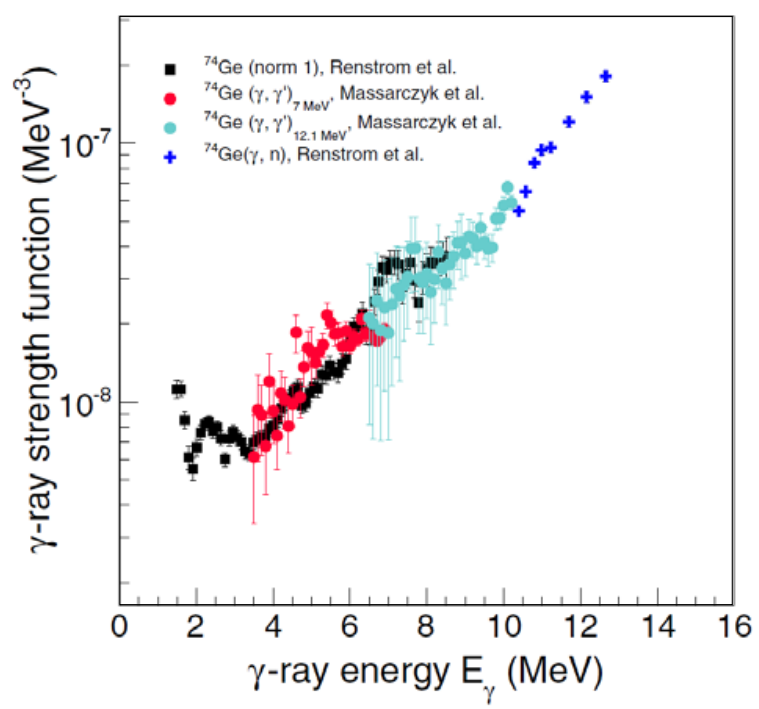

Figure 2. ${ }^{74}$ Ge photon strength function from the Oslo method (black squares), compared to that of photon scattering reactions (red and azur data), and from $(\gamma, n)$ reactions (blue crosses) [7].

\section{Results}

\section{1. ${ }^{74} \mathrm{Ge}$}

For ${ }^{74} \mathrm{Ge}$ three relevant data sets are now available from the reactions ${ }^{74} \mathrm{Ge}\left({ }^{3} \mathrm{He},{ }^{3} \mathrm{He}^{\prime}\right)[7]$ and ${ }^{74} \mathrm{Ge}\left({ }^{4} \mathrm{He},{ }^{4} \mathrm{He}^{\prime}\right)$ [8], and ${ }^{74} \mathrm{Ge}\left(\gamma, \gamma^{\prime}\right)$ [9], which were obtained through an international effort of several institutes. The results from ${ }^{74} \mathrm{Ge}\left(\gamma, \gamma^{\prime}\right)[9]$ and ${ }^{74} \mathrm{Ge}\left({ }^{3} \mathrm{He},{ }^{3} \mathrm{He}^{\prime}\right)$ [7] data show general good agreement (Fig. 2). However, the ${ }^{74} \mathrm{Ge}\left({ }^{3} \mathrm{He}^{3} \mathrm{He}^{\prime}\right)$ data show an increase in strength at $\mathrm{E}_{\gamma} \sim 7 \mathrm{MeV}$, while the uncertainties of the $\left(\gamma, \gamma^{\prime}\right)$ data are too large to interpret or confirm this increase in the PSF. The ${ }^{74} \mathrm{Ge}\left(\alpha, \alpha^{\prime}\right)$ experiment [8] was performed at iThemba LABS with the AFRODITE $\gamma$-ray detector array in conjunction with two identical particle-telescopes, each of them consisting of two segmented silicon detectors (in $\Delta \mathrm{E}-\mathrm{E}$ configuration) with an angular range of 20 to 72 degrees in the laboratory frame of reference. The beam with energy of
$47 \mathrm{MeV}$ impinged on a $500 \mu \mathrm{g} / \mathrm{cm}^{2}$ thick ${ }^{74} \mathrm{Ge}$ target to populate excited states in the inelastic scattering reaction.

AFRODITE, at the time of the experiment, consisted of nine Clover HPGe detectors with four detectors at 135 and five at 90 degrees mounted at a distance of $\sim 20 \mathrm{~cm}$ from the target. XIA digital electronics were used to acquire timestamped online data in singles mode. From these timestamped data, events with single, double, and higher fold coincidences were constructed. From double fold events, the $\alpha-\gamma$ coincidences were extracted by placing a gate on the $\alpha$-particles in the particle identification spectrum. The selection of correlated events was made with a coincidence time of less than $140 \mathrm{~ns}$ and uncorrelated event contributions were subtracted from the data by placing off-prompt time gates to the early and late sides of the prompt timing peak.

Transitions $\mathrm{E}_{\gamma}$ to the ground state were extracted with the condition $\left|E_{\gamma}-E_{x}\right| \leq 130 \mathrm{keV}$, which is the resolution of the particle telescope, where $\mathrm{E}_{x}$ refers to the excitation energy of the decaying state and is determined from the energy of the scattered $\alpha$ particles. This energy requirement, together with the differences in kinematic properties ensures that only transitions from ${ }^{74} \mathrm{Ge}$ are extracted, eliminating contributions due to contaminants.

Various combinations of angles between the direction of the recoiling nuclei and the $\gamma$-rays were used for the determination of angular distributions.

The spectrum of direct transitions to the ground state shows many states for $\mathrm{E}_{x}<6 \mathrm{MeV}$, and a high concentration of states/strength is also observed for $6.5<\mathrm{E}_{x}<8 \mathrm{MeV}$. Although the overall sensitivity to high-energy transitions is relatively poor, many transitions observed from $\left(\gamma, \gamma^{\prime}\right)$ data [5] could be observed. The unresolved, underlying $\gamma$-ray strength for $6.5<\mathrm{E}_{x}<8 \mathrm{MeV}$ is $\sim 50 \%$. Comparison with the most recent $\left(\gamma, \gamma^{\prime}\right)$ measurement [5] reveals several states, which were not populated in the $(\alpha, \alpha \gamma)$ reaction (see Fig. 3).

The multipole nature of the high-energy transitions was determined through angular distribution measurements, where the entire strength for $6.5<\mathrm{E}_{x}<8 \mathrm{MeV}$ was used simultaneously. For comparison, angular distributions of known dipole and quadrupole transitions were also extracted. The data support the overall dipole nature of the high-energy transitions and with natural-parity states being preferentially populated in this reaction, an assignment of spin-parity $\mathrm{J}^{\pi}=1^{-}$to the decaying states is made.

The comparison between $\left(\gamma, \gamma^{\prime}\right)$ and $(\alpha, \alpha \gamma)$ data shows the presence of two different regions in the energy range of the investigated dipole states. In the lower part $\left(3<\mathrm{E}_{x}<6 \mathrm{MeV}\right)$ the excitations due to $(\alpha, \alpha \gamma)$ are enhanced compared to the upper part $\left(6<\mathrm{E}_{x}<9 \mathrm{MeV}\right)$. For $\left(\gamma, \gamma^{\prime}\right)$ excitations, the trend is reversed indicating a dominant isovector nature of the higher-energy dipole states. The current result shows that many of the dipole states in the $6<\mathrm{E}_{x}<9 \mathrm{MeV}$ range in ${ }^{74} \mathrm{Ge}$ are mixed with larger isovector components. These results indicate that the dipole states in ${ }^{74} \mathrm{Ge}$ for $\mathrm{E}_{x}>6 \mathrm{MeV}$ do show the common Pygmy resonance scenario of dipole states splitting into two distinct parts: one at lower energy, whose states have a strong isospin mixing, and one at higher energy with predominant isovector character [10]. 


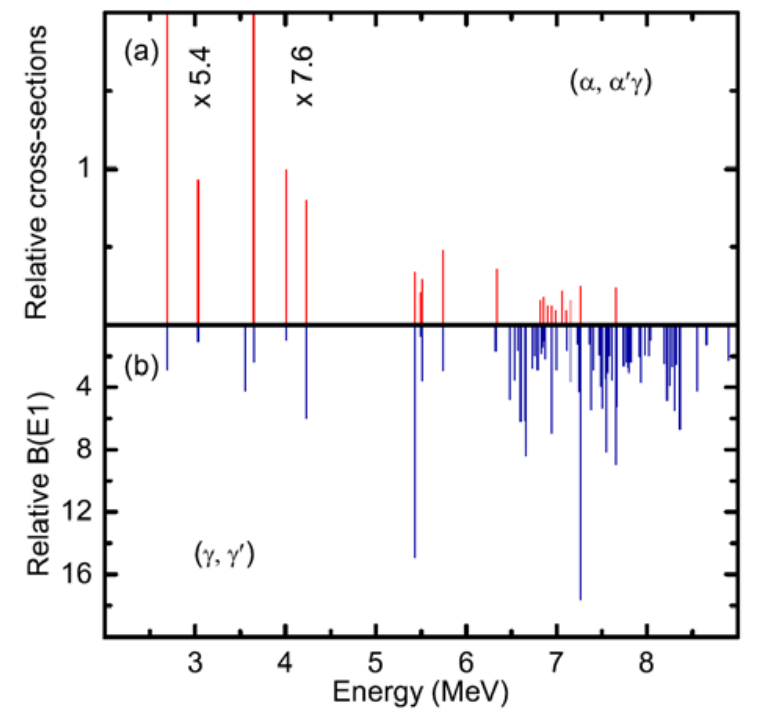

Figure 3. Top panel: relative cross-sections of E1 transitions from the $(\alpha, \alpha \gamma)$ data [8]. Bottom panel: relative B(E1) strength from $\left(\gamma, \gamma^{\prime}\right)$ data [9]. Numbers next to some transitions indicate the total value of relative cross section.

\section{2. ${ }^{95} \mathrm{Mo}$}

${ }^{95} \mathrm{Mo}$ is a nucleus that has received significant experimental attention over the years. At iThemba LABS an analysis on the $\gamma$-ray decay of neutron unbound states was performed [11]. This analytical work is based on a measurement that was carried out at the Lawrence Berkeley National Laboratory, where ${ }^{95}$ Mo was populated in the ${ }^{94} \mathrm{Mo}(\mathrm{d}, \mathrm{p} \gamma)$ reaction at a beam energy of $11 \mathrm{MeV}$, which has also led to results related to the PSF in ${ }^{95} \mathrm{Mo}$ [12] and to $(n, \gamma)$ cross sections from the surrogate approach [13]. The STARS-LIBERACE detector array [14] was used, consisting of BGO Compton-suppressed Clover-type and annular silicon detectors. Two Clover detectors were placed at 40, one at 90, and two at 140 degrees relative to the beam direction, at a distance of $20 \mathrm{~cm}$ from the target.

A particle telescopes was mounted downstream of the target with an angular coverage of 28 to 56 degrees. Particle- $\gamma$ coincidences of $\gamma$-ray multiplicity one or greater were analysed with a coincidence window of $100 \mathrm{~ns}$ between the $\gamma$ and proton events.

Proton-energy spectra in coincidence with a wellknown $\gamma$-ray transition de-exciting a discrete level were utilized. This type of spectrum allows for a clear identification of the excitation energy, directly populated in the $(\mathrm{d}, \mathrm{p})$ reaction, and the subsequent decay (directly or via a $\gamma$-ray cascade) to a lower-lying level.

The spectra of proton energies, gated on a specific $\gamma$-ray from low-lying discrete levels, are suitable for the determination of the spins of these levels. This determination can be accomplished by examining the behaviour of the energy dependence of the proton spectra just above the neutron separation energy $S_{n}$, as shown in Fig. 4. The energy dependence of the number of counts in the proton spectra above $S_{n}$ depends on the spins of lowlying levels. This effect has been observed previously and was interpreted as the influence of the centrifugal barrier on the emission of neutrons from unbound states [15]. However, its application to assigning spin to a low-lying level has not been discussed.

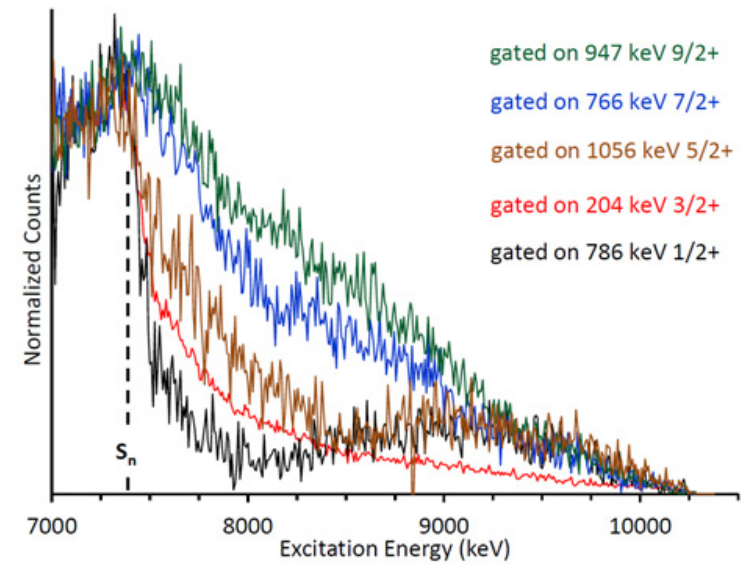

Figure 4. Proton-energy spectra in the vicinity of the neutron separation energy $S_{n}$, gated on low-lying discrete transitions. The increase in the proton signals, peaking at $\sim 9 \mathrm{MeV}$ is due to contributions in the $\gamma$-ray gates from $\left(\mathrm{n}, \mathrm{n}^{\prime}\right)$ reactions on the ${ }^{72} \mathrm{Ge}$ and in ${ }^{76} \mathrm{Ge}$ isotopes in the Clover crystals, respectively. The different spectra in this figure were normalized just below $S_{n}$.

The different shapes of the proton spectra above $S_{n}$ are due to (i) different ratios of $\gamma$ and neutron emission probabilities from levels with different spins produced above $S_{\mathrm{n}}$ (ii) high-level density in the region around $S_{\mathrm{n}}$, and (iii) low $\gamma$-ray multiplicity for feeding low-lying levels, which is responsible for the different distributions of spins for levels above $S_{n}$ that participate in feeding the low-lying levels. These conditions are combined with the fact that the initial distribution of spins of levels populated in the (d,p) reaction is rather broad. Both positive- and negative-parity states are expected to be populated with very similar cross sections.

The different $\gamma$ - and neutron-emission probabilities for levels with different spins are a consequence of the relatively high energies of the lowest-excited states in ${ }^{94} \mathrm{Mo}$. This implies that the probability for emission of a neutron from ${ }^{95} \mathrm{Mo}$ significantly decreases with spin of the level just above $S_{n}$, as it is not easy to emit neutrons with high angular momenta to populate the low-lying, low-spin states in ${ }^{94} \mathrm{Mo}$.

The decay paths of levels directly populated in the $(\mathrm{d}, \mathrm{p})$ reaction above $\mathrm{S}_{\mathrm{n}}$ to specific low-lying levels is complicated, but our experimental data indicate that a "memory" of initial spin is retained.

\section{Discussion}

\section{1. ${ }^{74} \mathrm{Ge}$}

The results indicate the suppression in relative cross section for the excitation of the Pygmy dipole resonance states in ${ }^{74} \mathrm{Ge}$, populated through inelastic $\alpha$-scattering, compared to photon scattering data for $\mathrm{E}_{x}>6 \mathrm{MeV}$. The observed dipole response splits into two distinct parts: one at lower energy, whose states have a strong isospin mixing and one at higher energy with predominant isovector character, consistent with previously observed Pygmy dipole resonance behaviour. Although, the ${ }^{74} \mathrm{Ge}\left(\gamma, \gamma^{\prime}\right)$ [9] and ${ }^{74} \mathrm{Ge}\left({ }^{3} \mathrm{He},{ }^{3} \mathrm{He}^{\prime}\right)$ [7] data exhibit overall agreement of the PSF, the existence and underlying mechanism of the enhanced strength at $7 \mathrm{MeV}$ could not be resolved from these measurements alone. The results from this 
${ }^{74} \mathrm{Ge}(\alpha, \alpha \gamma)$ work not only confirm the existence of the additional strength at $7 \mathrm{MeV}$ but also establish its physical mechanism to be consistent with that of a typical Pygmy dipole resonance.

\section{2. ${ }^{95} \mathrm{Mo}$}

The spacing of the lowest excited states in ${ }^{94} \mathrm{Mo}$ leads to a significant suppression of neutron emission from high-spin levels populated just above $S_{n}$. This effect is responsible for the strong spin dependence in the population of low-lying levels in ${ }^{95} \mathrm{Mo}$. Utilizing data from proton- $\gamma$ coincidences, these differences can be used to determine the spins of low-lying levels which is a complementary technique to other spin-determining methods. It also provides insight into the detailed correction factor for each spin that is needed for $(\gamma, \mathrm{n})$ measurements, as the competition of $(\gamma, \mathrm{n})$ and $\left(\gamma, \gamma^{\prime}\right)$ reactions increases towards $S_{n}$. Based on these results, the $(\gamma, n)$ correction has been performed in ${ }^{95}$ Mo to normalize PSF data below $\mathrm{S}_{\mathrm{n}}$ to $(\gamma, \mathrm{n})$ data [16]. The correction is significant and has the potential to effect the normalization if not taken into account. This highlights the importance of accounting for angular momentum barrier effects when normalizing PSF data in the vicinity of $S_{n}$.

\section{Conclusion}

A single measurement of the statistical properties of a nucleus cannot address all the features of the PSF. The work on ${ }^{95} \mathrm{Mo}$ and ${ }^{74} \mathrm{Ge}$ highlights the importance to perform and combine results of many measurements on a given nucleonic system to obtain and understand the "global" PSF. Having a wealth of data available is particularly important for nuclei where different measurements do not agree with each other. This will likely be the only successful approach to unravel the underlying reasons of data inconsistencies. While ${ }^{74} \mathrm{Ge}$ and some of the Mo isotopes already provide an acceptable amount and variety of measurements, other nuclei, in particular those exhibiting significant disagreement between measurements, need to be identified and measured. These nuclei can be envisioned to become cases on which the community focuses their effort to obtain the best possible knowledge of the PSF and underlying resonance features. This envisioned approach is consistent with the recently initiated IAEA CRP on "Updating Photonuclear Data Library and Generating a Reference Database for Photon Strength Functions" [17].

This work is supported by the National Research Foundation of South Africa grant Nos. 92789, 83867, 75998, the Norwegian Research Council, the LLNL U.S. DoE contract No. DE-AC5207NA27344, the DoE NNSA through the Nuclear Science and Security Consortium under DE-NA-0003180, and the IAEA under Research Contract 20454.

\section{References}

[1] R. Schwengner, 2nd Workshop on Nuclear Level Densities and Photon Strength, Oslo, Norway (2011)

[2] M. Krtička, F. Bečvář, Eur. Phys. Journal, Web of Conferences 2, 03002 (2010)

[3] H. Utsunomiya et al., Phys. Rev. C 88, 015805 (2013)

[4] A. Schiller et al., Nucl. Instrum. Meth. Phys. Res. A 447, 498 (2000)

[5] R. Schwengner et al., Phys. Rev. C 78, 064314 (2008)

[6] https://www-nds.iaea.org/exfor/servlet (retrieved 2016)

[7] T. Renstrøm et al., Phys. Rev. C 93, 064302 (2016)

[8] D. Negi et al., Phys. Rev. C 94, 024332 (2016)

[9] R. Massarczyk et al., Phys. Rev. C 92, 044309 (2015)

[10] D. Savran, T. Aumann, and A. Zilges, Prog. Part. Nucl. Phys. 70, 210 (2013)

[11] M. Wiedeking et al., Phys. Rev. C 93, 024303 (2016)

[12] M. Wiedeking et al., Phys. Rev. Lett. 108, 162503 (2012)

[13] B.L. Goldblum et al., Phys. Rev. C 85, 054616 (2012)

[14] S.R. Lesher et al., Nucl. Instr. Meth. Phys. Res. A 621, 286 (2010)

[15] G. Stengl et al., Nucl. Phys A290, 109 (1977)

[16] M. Krtička et al., Phys. Rev. C 93, 054311 (2016)

[17] IAEA INDC(NDS)-0712 (2016). https://wwwnds.iaea.org/publications/indc/indc-nds0712.pdf 